\title{
Quality of Service Support for ODMRP Unicast Routing in Ad hoc Networks
}

\author{
Amir Darehshoorzadeh \\ Computer Engineering \\ Department, Iran University of Department, Iran University of \\ Science and Technology \\ Tehran, Iran \\ E-mail: darehshoori@,comp.iust.ac.ir \\ M.Reza Jahed Motlagh \\ Computer Engineering \\ Science and Technology \\ Tehran, Iran \\ E-mail : jahedmr@iust.ac.ir
}

\author{
Mehdi Dehghan \\ Computer Engineering \\ Department, Amirkabir \\ University of Technology \\ Tehran, Iran \\ E-mail : dehghan@ce.aut.ac.ir
}

\begin{abstract}
An ad hoc network is comprised of mobile hosts without any wired infrastructure support. The primary concerns in ad hoc networks are bandwidth limitation and unpredictable dynamic topology. Therefore, efficient bandwidth utilization is crucial in routing protocols. The On-Demand Multicast Routing Protocol (ODMRP) was designed for multicast routing in adhoc networks. However, ODMRP supports unicast routing too. It is very important to efficiently allocate and consume link bandwidth in this protocol, especially when many sessions are working concurrently. In this paper, we propose a new technique for supporting QoS Routing in ODMRP using good estimation of available and required bandwidth. Simulation results show that using QoS routing for ODMRP in unicast mode improves network performance in presence of mobility, by searching for suitable paths.
\end{abstract}

\section{Introduction}

An ad hoc network is a dynamically reconfigurable wireless network with no fixed wired infrastructure. Each node can function both as a network host for transmitting and receiving data and as a network router for routing packets to the other nodes. Ad hoc networks have numerous practical applications such as military applications, emergency operations, and wireless sensor networks. In many applications, ad hoc networks carry diverse multimedia applications such as voice, video and data. In order to provide quality delivery to delay sensitive applications such as voice and video it is imperative that ad hoc networks provide quality of service (QoS) support in terms of bandwidth and delay [6].

Quality of Service (QoS) is the performance level of a service offered by the network to the user. After receiving a service request from the user, the first task is to find a suitable loop-free path from the source to the destination that will have the necessary resources available to meet the QoS requirements of the desired service. This process is known as QoS routing. After finding a suitable path, a resource reservation protocol is employed to reserve necessary resources along that path [7].QoS routing protocols search for routes with sufficient resources in order to satisfy the QoS requirement of a flow. The information regarding the availability of resources is managed by a resource management module, which assists the QoS routing protocol in its search for QoS feasible paths.

The On Demand Multicast Routing Protocol (ODMRP) is designed for Ad hoc networks [8]. It can function as both multicast and unicast as well as coexist with any unicast routing protocol [9]. Other adhoc multicast routing protocols such as Ad hoc Multicast Routing (AMRoute) [10], Core Assisted Mesh Protocol (CAMP) [11], Lightweight Adaptive Multicast (LAM) [12] and Reservation Based Multicast (RBM) [13] must run on top of a unicast routing protocol. CAMP, RBM and LAM in particular, only work with certain underlying unicast protocols.

In this paper, we propose a new technique for supporting QoS Routing for ODMRP in unicast mode, using good estimation of available and required bandwidth. The reminder of this paper is organized as follows. Section 2 describes unicast functionality of ODMRP. Section 3 describes admission control and calculation of available and consumed bandwidth. Section 4 describes our new approach for QoS in ODMRP. Section 5 follows with the simulation results and concluding remarks are made in section 6.

\section{ODMRP Mechanism in Unicast Mode}

ODMRP builds and maintains routes on demand by the source. Similar to other on-demand unicast routing protocols, a query phase and a reply phase comprise 
the protocol. When a source node has data to send but no route information to that destination is known, it floods a JOIN QUERY packet with a piggybacked data payload. When a node receives a non-duplicate JOINQUERY, it stores the last hop node information in its route table (i.e., backward route) and rebroadcasts the packet. This process is continued until JOIN QUERY reaches the destination. The destination replies back to the source via the selected route with JOIN QUERY. When a node receives a JOIN REPLY, it checks if the next node address of JOIN REPLY entry matches its own address. If it does, the node realizes that it is on the path to the source. It then sets its forwarding flag for this session and broadcasts its own JOIN REPLY built upon matched entry. The previous node address is obtained from the route table where the entry was recorded when JOIN QUERY was received. The JOIN REPLY is propagated until it reaches the source node. Intermediate nodes that relay JOIN REPLY mark themselves as forwarder of this session.

This process constructs the route from the source to the destination. After these processes, source can transmit data packets to receiver via selected route and forwarder nodes. Periodic JOIN QUERIES are sent only when source has still data to send. Figure 1 depicts the route <S-B-C-E-D $>$ establishment procedure. When receiving a data packet, a node forwards the packet only if it is not a duplicate and the forwarding flag of that session has not expired. This procedure minimizes traffic overhead and prevents sending data packets through stale routes [4]. In this protocol, nodes don't need any explicit packets to leave a session. If a sender wants to leave a session, it stops sending JOIN QUERY packets since it doesn't have any data to send. If a receiver wants to leave a session it stops sending JOIN REPLY to the source and remove the address of session from its member table. Forwarder nodes of a session change their state to non-forwarding if not refreshed before they timeout.

\section{Admission control}

To provide QoS requirements on a path, the admission control policy should guarantee the requested minimum bandwidth $B_{\min }$ for each flow. Bandwidth reservation by admission control is made at every node in the route setup phases, based on calculation as described in the next section.

\subsection{Bandwidth control}

To determine if there is enough bandwidth for a new flow $J$, all we need to know is the available link capacity and the bandwidth to be consumed by the flow. Because of shared medium, a node can successfully

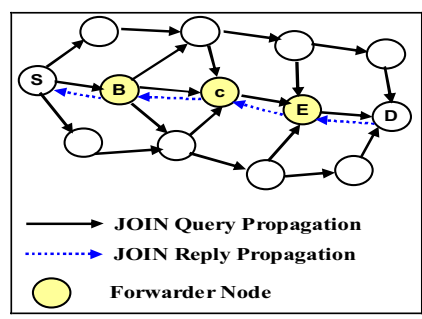

Figure 1. On-Demand Procedure for Route Setup in ODMRP use the channel only when all its neighbors do not transmit and receive packets at the same time [6]. As illustrated in [6] available bandwidth at node $I$ can be given by:

$B_{\text {available }}(I)=B-\sum_{j \in N(I)} B_{\text {self }}(j)$.

Where $B$ is the raw data rate of the node $I$ and $B_{\text {self }}(j)$ is the total traffic between node $j$ and its neighbors, i.e., the bandwidth consumed by the traffic transmitted or received by node $j$. Given the requested bandwidth $B_{\text {min }}$, the bandwidth to be reserved for the flow $j$ at node $I$ is:

$$
B_{I}(j)= \begin{cases}B_{\min } & \text { if source or destination } \\ 2 B_{\min } & \text { else }\end{cases}
$$

Since the intermediated nodes need to receive and forward flow $j[6]$.

As illustrate in [6] bandwidth that is consumed for flow $j$ on node $I$ 's channel can be given by:

$$
B_{\text {consumed }}(I, j)=B_{\text {uplink }(I)}(j)+B_{\operatorname{downlink}(I)}(j)
$$

Where $B_{\text {uplink (I) }}(j)$ is the reserved bandwidth for flow $j$ on the upstream neighbor of node $I$ and $B_{\operatorname{downlink}(I)}(j)$ is the bandwidth that the downstream neighbor of node $I$ reserved for flow $j$. Note that $B_{\text {uplink }(I)}(j)$ and $B_{\operatorname{downlink}(I)}(j)$ can either equal to $B_{\min }$ or $2 B_{\min }$ as shown in (2). By comparing the value of $B_{\text {available }}(I)$ and $B_{\text {consumed }}(I, j)$, each node can now decide whether to accept the flow or not [6].

\section{QOS-ODMRP Unicast Mechanism}

\subsection{Neighborhood maintenance}

Neighbor information is very important in QOSODMRP since it provides the local topology and traffic information. To maintain the neighborhood information, every node in the network is required to periodically send out a HELLO message to its neighbor that announced its existence and its traffic information. Each node $I$ insert its self traffic $\left(B_{\text {self }}(I)\right)$ in its HELLO packet. HELLO packets are sent at a default rate of one packet per two seconds, with time to live (TTL) set to 1 . When a node receives a HELLO packet from its neighbor, it stores or updates the information of that neighbor in its neighbor list $N(I)$. Failure to 
receive any packet from a neighbor for a certain period of time is taken as indication that the link to that neighbor is down.

\subsection{Route Setup}

Similar to ODMRP, When a source has data to send but no route information to destination is known; it appends the requested bandwidth for this session $\left(B_{\text {min }}\right)$ to the JOIN QUERY packets and floods it. When a node receives a non-duplicate JOIN QUERY, it checks whether it has enough bandwidth for this request or not. For this purpose it computes and compares the channel's $B_{\text {Available }}$ and $B_{\text {Consume }}$ of that flow. If $B_{\text {Available }}$ is greater than $B_{\text {Consume }}$ and also greater than the minimum bandwidth to be reserved $\left(B_{\min }\right.$ or $2 B_{\text {min }}$ ) this node will adds a new entry in its reserve table with status explored as shown in Figure 2. It also stores the last hop node information in its route table (i.e., backward route) and rebroadcasts the packet. The node will only remain in explored status for one second. If no reply arrives at the explored node, in time, the entry in reserve table will be ignored. Since the node that propagates JOIN QUERY do not aware of downstream node in the route to the destination, we used $B w_{\text {Uplink }}$ as the estimation of $B_{\text {Consume }}$ for propagating JOINQUERY packet. The destination replies back to the source via the selected route with JOIN QUERY. When a destination receives a JOIN QUERY packet it builds the JOIN REPLY packet and if it has enough bandwidth for this session it broadcasts the JOINREPLY packet to the upstream node and updates the reserve table status to registered (figure 3). After registration, the nodes are ready to accept the real data packets of the flow. The node will only stay in registered status for one second, if no data packet arrives at the registered node in time; the entry in reserve table is ignored. When a node receives a JOINREPLY, it checks if the next node address of its entry matches its own address. If matched, the node realizes that it is on the path to the source. It checks its available bandwidth and compares it with $B_{\text {Consume }}$ of this flow. If $B_{\text {Available }}$ is greater than $B_{\text {Consume }}$ it then sets its forwarding flag for this session and broadcasts its own JOIN REPLY built upon matched entry. The previous node address is obtained from the route table where the entry was recorded when JOIN QUERY was received. The JOIN REPLY is propagated until it reaches the source session. Intermediate nodes that relay JOIN REPLY mark itself as forwarder of this session. As we explained before when node handles JOIN QUERY it only aware of its upstream node and used $B w_{\text {Uplink }}$ as the estimation of $B_{\text {Consume }}$, but after receiving JOIN REPLY it aware of its downstream node and can calculate the precise $B_{\text {Consume }}$ for this session. This bandwidth availability rechecking is essential to reduce transient routes construction.

\subsection{Data Forwarding}

After constructing the route, a source can send packets to the destination via selected route and forwarding nodes. After receiving the data packet, a node forwards it only when it is not a duplicate packet and the forwarding flag for this session has not expired and also if the status of reserved bandwidth entry is registered or reserved. If status of its reserved bandwidth entry is registered, it changes it to reserved status and sends data (Figure 4). The node will only stay in reserved status until next JOIN QUERY send. This procedure minimizes the traffic overhead and prevents sending packets through stale routes. As shown in Figure 2 suppose node $C$ and $E$ have not enough bandwidth for session that source node $S$ wants to establish, thus they do not relay JOIN QUERY and the established route is $S-F-G-H-I-D$ (Figure 3) but in ODMRP, although the route $S-B-C-E-D$ could not provide enough bandwidth, it may be selected as an active path. Another example is shown in Figure 5. Suppose there is a session between $S_{l}$ as a sender and node $B$ as a receiver, this session needs at least $512 \mathrm{~kb} / \mathrm{s}$ bandwidth to be reserved at each node and channel capacity of each node is $2 \mathrm{Mbit} / \mathrm{sec}$. Node $S_{2}$ tries to establish a new path toward node $C$ through $A$. if this new session also needs $512 \mathrm{~kb} / \mathrm{s}$ of bandwidth, without any mechanism for bandwidth management in ODMRP, although none of node $A$ and $C$ has enough bandwidth for this new flow $\left(S_{2}\right)$ but the path will be constructed and affected the previous session $\left(S_{1}\right)$. But in QoS-ODMRP each node compares $B_{\text {Available }}$ and $B_{\text {consume }}$ and when there isn't sufficient bandwidth for new session it will be rejected.

\subsection{Route Maintenance}

QoS-ODMRP uses soft state for route maintenance. Each source periodically sends out JOIN QUERY packet,

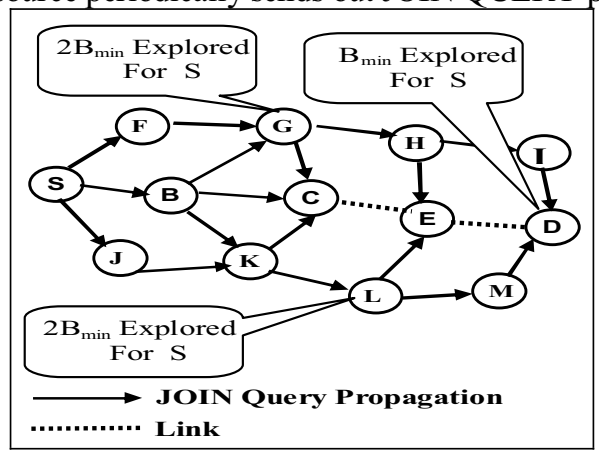

Figure 2. JOIN Query propagation in QOS-ODMRP 


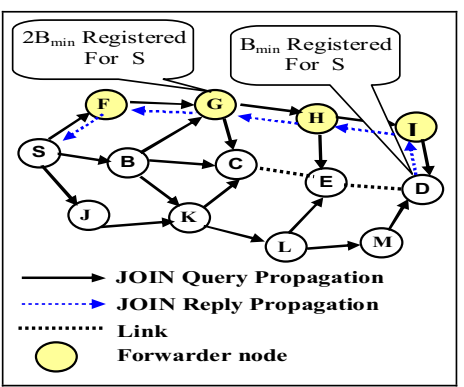

Figure 3. Route setup with JOIN Reply in QOS-ODMRP

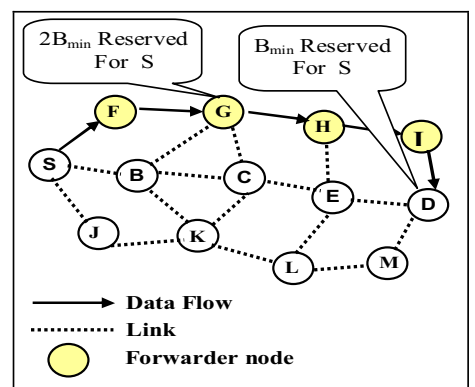

Figure 4. Data flow changes registered status to reserved

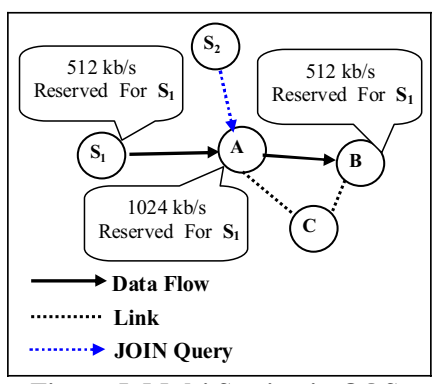

Figure 5. Multi Session in QOS-

ODMRP as well as route setup procedure, to refresh or repair break routes. JOIN REPLY packets are backed through reverse routes and forwarding nodes are updated.

\subsection{Soft State}

In QoS-ODMRP, no explicit control packets need to be sent to join or leave the session. If a source wants to leave the session, it simply stops sending JOINQUERY packet, since it does not have any data to send. If a destination no longer wants to receive from its source, it does not send JOIN REPLY packet for that source. Forwarder nodes are demoted to nonforwarding if not refreshed (no JOIN REPLY received). When a node leaves a session or is demoted to non-forwarding, it releases the requested bandwidth for that session and sends a HELLO packet to its neighbor to inform them about its new self traffic.

\section{Performance Evaluation}

\subsection{Simulation Environment}

The simulator was implemented within the Global Mobile Simulation (GloMoSim) library [1].The GloMoSim library is a scalable simulation environment for wireless network system using the parallel discrete-event simulation capability provided by PARSEC [2].Our simulation modeled a network of 50 mobile hosts placed randomly within a $1000 \times 1000 \mathrm{~m}^{2}$ area. Radio propagation range for each node was 250 meters and channel capacity was $2 \mathrm{Mbit} / \mathrm{sec}$. Each simulation executed for 600 seconds of simulation time. The IEEE 802.11 Distributed Coordination Function (DCF) [3] was used as the medium access control protocol. We used Constant Bit Rate as our traffic since the GloMoSim doesn't support Variable Bit Rate traffic (VBR). The size of data payload was 512 bytes. We used Random-Way point as mobility model that nodes randomly selected the moving direction, and when they reached the simulation terrain boundary, they bounced back and continued to move.

\subsection{Methodology}

To evaluate the unicast performance of QOSODMRP, we simulated and compared the following schemes:

- ODMRP (On-Demand Multicast Routing Protocol)

- QOS-ODMRP (Quality of Service On-Demand Multicast Routing Protocol)

We evaluated both schemes as a function of speed. The number of data sessions was set to 5 and 10 , and speed was varied from 0 to $72 \mathrm{Km} / \mathrm{hr}$ and pause-time was 3 seconds. Sessions were established with interval of 5 seconds and remained until end of simulation time. To evaluate the performance of our protocol each sources sent data as rate of $64 \mathrm{Kbit} / \mathrm{s}$ and $128 \mathrm{Kbit} / \mathrm{s}$. we collected the following metrics:

- Packet Delivery Ratio: The number of data received by destinations over the number of data sent by sources.

- Number of total packets transmitted per data packet delivered: The number of all packets (data and control packets) transmitted divided by the number of data packet delivered to the destinations.

- Traffic admission ratio: ratio between the number of data packets sent to the network from the sources and the number of data packets generated at the sources.

\section{Simulation Results}

\subsection{Packet Delivery Ratio}

The packet delivery ratios as a function of mobility speed are shown in Figures 6 to 9. We can observe that as speed increases because of links break the packet delivery ratios decrease in both protocols As the traffic rate increases $(64 \mathrm{~kb} / \mathrm{s}$ to $128 \mathrm{~kb} / \mathrm{s})$ the data delivery ratio of ODMRP is decreased about $35 \%$ but in the case of QoS-ODMRP the data delivery ratios degrade less than $10 \%$.

Also the data delivery ratios of ODMRP decrease significantly as the number of sessions increase 
because of collision accusers. But in our protocol, QoS-ODMRP, by utilizing efficient bandwidth and don't accept all sessions at all simulation scenarios, data delivery ratios are significantly higher than ODMRP.

\subsection{Number of Total Packets Transmitted per Data Packet Delivered}

Figures 10 to 13 show control and data overhead over data received for two protocols in presence of mobility. Although QoS-ODMRP uses extra control packet (HELLO message) but by using this control packets, QoS-ODMRP prevents propagation of JOINQueries and JOIN Replies when there isn't enough bandwidth for a session therefore, its control and data overhead over data received is much low than ODMRP.

\subsection{Traffic Admission Ratio}

The rest figures show the traffic admission ratio for QoS-ODMRP means that the traffic of accepted

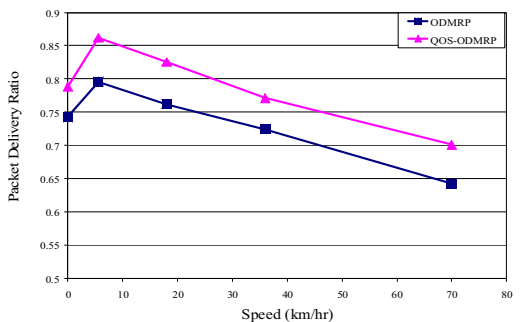

Figure 6. Packet Delivery Ratio (5 Sessions, $C B R=64 \mathrm{~Kb} / \mathrm{s}$ )

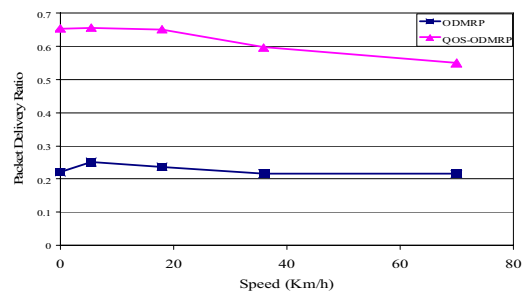

Figure 9. Packet Delivery Ratio (10 Sessions, CBR=128Kb/s)

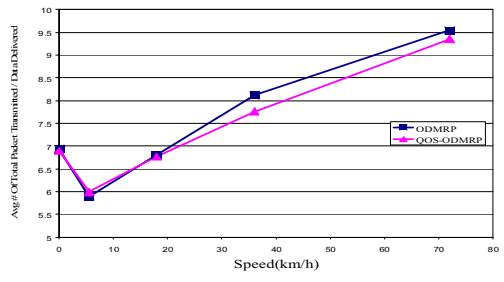

Figure 12. Number of Total Packets Transmitted per Data Packet Delivered

$(10$ Sessions, $\mathrm{CBR}=64 \mathrm{~Kb} / \mathrm{s})$

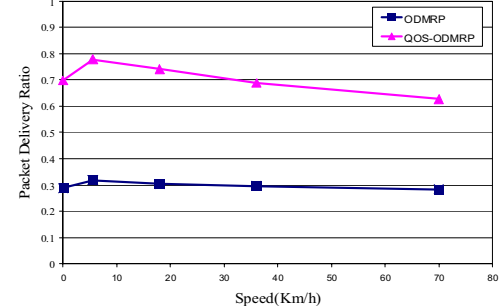

Figure 7. Packet Delivery Ratio (5 Sessions, $\mathrm{CBR}=128 \mathrm{~Kb} / \mathrm{s}$ )

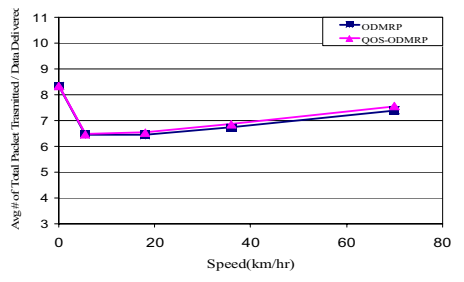

Figure 10. Number of Total Packets Transmitted per Data Packet Delivered

(5Sessions, $\mathrm{CBR}=64 \mathrm{~Kb} / \mathrm{s}$ )

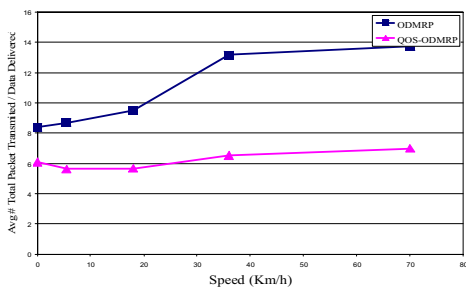

Figure 13. Number of Total Packets Transmitted per Data Packet Delivered

(10 Sessions, $\mathrm{CBR}=128 \mathrm{~Kb} / \mathrm{s})$

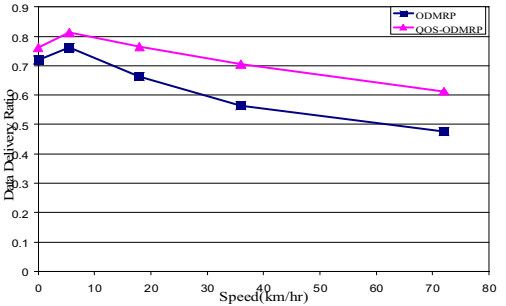

Figure 8. Packet Delivery Ratio (10 Sessions, $\mathrm{CBR}=64 \mathrm{~Kb} / \mathrm{s}$ )

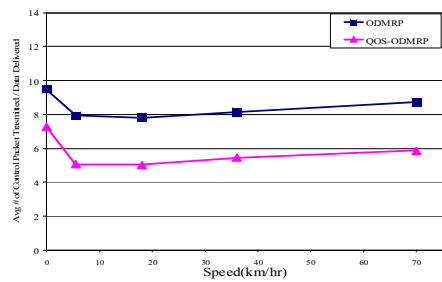

Figure 11. Number of Total Packets Transmitted per Data Packet Delivered

(5 Sessions, CBR=128Kb/s)

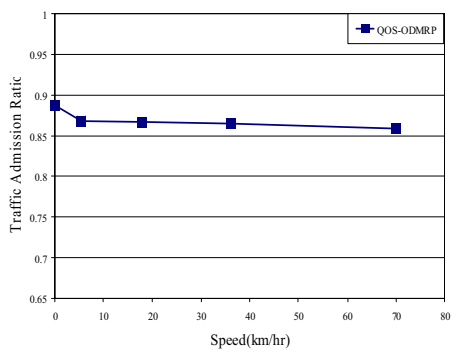

Figure 14. Traffic Admission Ratio (5Sessions, $\mathrm{CBR}=64 \mathrm{~Kb} / \mathrm{s})$ 


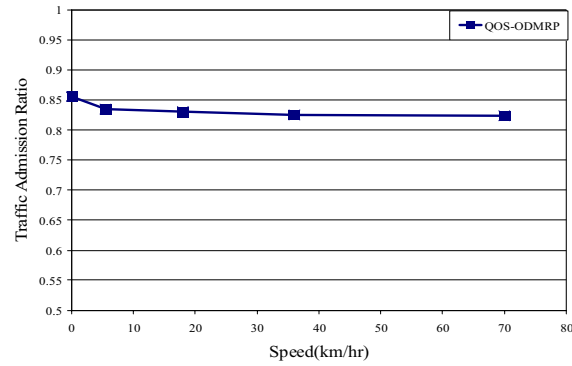

Figure 15. Traffic Admission Ratio (5 Sessions, $\mathrm{CBR}=128 \mathrm{~Kb} / \mathrm{s})$

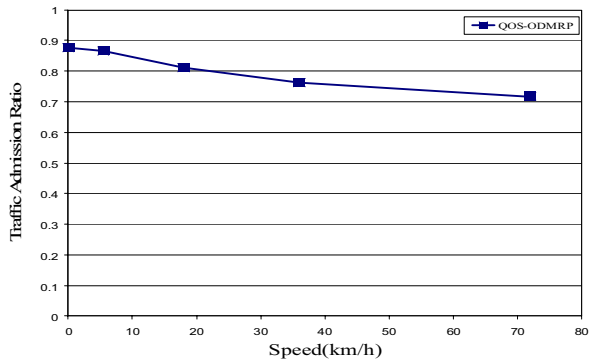

Figure 16. Traffic Admission Ratio $(10$ Sessions, $C B R=64 \mathrm{~Kb} / \mathrm{s})$

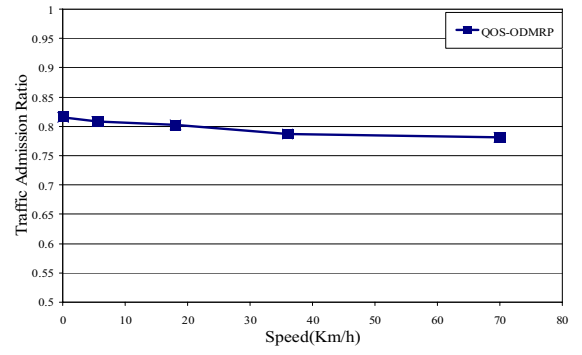

Fig 17: Traffic Admission Ratio (10 Sessions, CBR=128Kb/s)

\section{ACKNOWLEDGMENTS}

This work has been supported by Iran Telecommunication Research Center (ITRC).

\section{REFERENCES}

[1] R. Bagrodia, R. Mayer, M. Takai, Y.Chen, X. Zeng, J.Martin and H.Y.Song,PARSEC:A parallel simulation environment for complex systems, IEEE Computer 31(10)(October 1998) 77-85.

[2] UCLA Parallel Computing Laboratory and Wireless Adaptive Mobility Laboratory, GloMoSim:A scalable simulation environment for wireless and wired network system,http://pclcs.ucla.edu/projects/domains/glomosim.html. [3] IEEE Computer Society LAN MAN Standards Committee, Wireless LAN Medium Access Protocol (MAC) and Physical Layer (PHY) Specification, IEEE Std 802.111997. The Institute of Electrical and Electronics Engineering, New York, NY, 1997.

[4] S. Lee, W. Su, M. Gerla, "Wireless Ad hoc Multicast Routing with Mobility Prediction," Kluwer Academic Publishers. Manufacture in the Netherlands 2001, pp. 351360.

[5] w.su,lee and Gerla, "Mobility Prediction and Routing in Ad hoc Wireless Networks," International Journal of Network Management, Volume 11, Issue1, pp.3-30.

[6] Qi Xue and Aura Ganz, ad hoc QOS on-demand routing (AQOR) in mobile ad hoc networks, Journal of parallel and distributed computing 2003, pp 154-165.

[7] C. Siva Ram Murphy and B. S. Manoj, Ad Hoc Wireless Networks Architecture and Protocols, $1^{\text {st }}$ ed, Prentice hall, pp.505-580.

[8] S. Lee, M. Gerla, C. Chiang, "On-Demand Multicast Routing Protocol", Proceeding of IEEE WCNC 1999.

[9] S. Lee, W. Su, M. Gerla, "Exploiting the Unicast Functionality of the On-Demand Multicast Routing Protocol", Proceeding of IEEE WCNC 2000, Chicago, IL (September 2000).

[10] E.Bommaiah, M. Liu, A. McAuley, and R. Talpade, "AMRoute: Ad hoc Multicast Routing Protocol," internet draft, draft-talpade-manet-amroute-00.txt, August 1998.

[11] M. S. Corson and S. G. Batsell, "A Reservation - Based Multicast (RBM) Routing Protocol for Mobile Networks: Initial Route Construction Phase," ACM/Baltzer Journal of Wireless Networks (WINET), Vol.1, no.4, Desember 1995, pp.427-450.

[12] J. J. Garcia-Luna-Aceves and E. L. Madruga, "A Multicast Routing Protocol for Ad Hoc Networks," Proceedings of IEEE Conference on Computer Communications (INFOCOM), New York, NY, March 1999, pp.784-792.

[13] L. Ji and M. S. Corson, "A Lightweight Adaptive Multicast Algorithm," Proceedings of the IEEE Global Telecommunication Conference (GLOBECOM), Sydney, Australia, November 1998, pp. 1036-1042. 